\title{
RAZÃO E DESRAZÃO NAS TOMADAS DE DECISÃO
}

\author{
Álvaro Machado Dias'
}

Resumo:Tal como se apresenta na atualidade,o campo de Teorias de Tomadas de Decisão reflete a intersecção de três desenvolvimentos teóricos principais: Utilidade Esperada, Heurísticas e Desvios e Intuição Holística. As relações entre estes não são clarividentes, nem estão estabelecidas na literatura sobre o assunto, sobretudo porque algumas das tendências em jogo ainda são muito novas. Meu objetivo é contribuir para o suprimento desta lacuna, oferecendo uma visão geral do campo, particularmente sensível às demandas epistemológicas às quais cada novo desenvolvimento respondeu e às limitações destas respostas. De especial interesse é o fato de que isto irá habilitar o leitor a compreender os fundamentos do novo conceito de intuição decisional que desponta e a se posicionar criticamente em relação ao mesmo.

Palavras-chave: Tomadas de Decisão. Utilidade Esperada. Heurísticas e desvios. Intuição holística.

\section{Introdução}

O processo humano de tomada de decisões é um assunto que fascina os estudiosos da mente e do comportamento humano há tempos imemoriáveis. "Economistas", "psicólogos" e "cientistas cognitivos" avant la lettre, primeiro se verteram sobre aquele para só então darem escopo a estas designações, que agora nos permitem discriminar os ramos de especialização no estudo do fenômeno.

De maneira elementar, a tomada de uma decisão expressa o investimento sobre uma aparição mental, tradicionalmente representativa de algo que existe no mundo,

1 Agradeço à Prof. Dra. Emma Otta (PSE-IPUSP) pelas discussões críticas, sugestões e revisão atenciosa, ao Prof. Dr. Luiz Roberto G. de Britto (ICB-USP), pela orientação da pesquisa de doutorado (NEC-USP), do qual este artigo é parte, e ao economista Maracajaro Mansor pelas sugestões para tornar a discussão matemática mais intuitiva. Este artigo foi realizado com apoio da bolsa de doutorado do CNPQ. 
em detrimento de uma ou mais opções, que também devem ser representativas de um representante externo. Chamamos a estas existências de commodities e, assim, à vasta maioria das tomadas de decisões, de escoIha entre commodities. Estas podem ser comparáveis ou incomparáveis e preconizam um dos grandes temas em destaque no campo de análise de tomadas de decisão: o estabelecimento de valor.

Deixando terminantemente de lado o problema epistemológico fundamental representado pela relação representante-representação e, provisoriamente, também deixando de lado o problema do estabelecimento de valores, podemos notar que as coisas são um tanto mais complexas do que a descrição acima pode sugerir: decisões são maturadas interiormente em períodos que, mais do que simplesmente variáveis (de decisão em decisão), são indetermináveis em termos objetivos.

Disto se revela que a circunscrição de um processo decisional não pode ser estabelecida com rigor, tal como, mais amplamente, o recorte de um pensamento (na forma de verbalização proposicional) dentre a massa de pensamentos com que se associa, mediante a qual sempre carregará alguma dose de arbitrariedade.

Paralelamente, definir de antemão contingências mínimas capazes de circunscrever uma decisão é uma tarefa impossível. E, tal como nos revela o condenado à morte que decide pela injeção letal em relação à cadeira elétrica, tampouco é clarividente se a força de uma decisão está em seu efeito sobre o sujeito ou nas transformações que produz no mundo exterior. Mais amplamente: a perspectiva de ter que tomar uma decisão nos remete à de um investimento intencional de consistência incerta, apresentando-se já em sua faceta mais elementar, como um problema bidimensional: o que/ por que se decide e até que ponto se decide.

Dentre os sistemas filosóficos ocidentais em que este problema fezse presente, encontrou-se de sobremaneira conectado ao conceito de livrearbítrio e, naturalmente, às suas críticas deterministas, como, por exemplo, entre os estoicos, paradigmaticamente representados por Chrysippus, que se opunham pelo enfoque nas leis naturais da matéria, à assunção pitagórica de liberdade e que assim delinearam um debate paradigmático para o desenvolvimento subsequente do pensamento ocidental.

Muito em função do caráter inefável deste complexo debate, nos últimos dois séculos, a tendência na análise de tomadas de decisão passou a ser gradativamente definida pela assunção (não necessariamente deliberada) de um recorte epistemológico entre as questões ontológicas e a regulamentação pontual de escolhas, particularmente influenciada pela perspectiva liberal e utilitarista de John Stuart Mill (1963-1991), ativamente empenhado na discriminação de objetos e razões para a felicidade e assim para a escolha entre commodities.

Seja em termos de contingências exteriores, aspectos subjetivos, processos neurais e simulações computacionais, decidir se tornou quase 
sinônimo de "relação direta/indireta com o mundo exterior", conforme o moderno campo das análises de tomadas de decisão ganhou fôlego ao se voltar às questões cotidianas, em relação às quais, apenas recentemente vicejaram tendências mais consistentes à relativização dos paradigmas canônicos, âmbito no qual se inserem os modelos de heurística e intuição decisional.

Ainda é cedo para a realização de uma exegese deste estágio mais recente, mas podemos apontar um resgate contemporâneo de duas tendências relegadas a segundo plano pela tradição instaurada no campo de tomadas de decisão durante os primeiros três quartos do século XX: 0 compromisso em contemplar um modo de agir e pensar, no qual a racionalidade é uma esfera entre outras, e a influência da sensorialidade sobre os processos de escolha.

Quanto ao primeiro, este movimento produziu uma resposta estratégica à questão intencionalidade/livre arbítrio e se fiou às diversas modalidades de contraposição à hiperestasia do Cogito, sob a máxima de que os processos mentais, ou de sinalização cerebral, escoram-se no funcionamento de um endofenótipo cujo design não urge à perfeição, mas reflete as imposições reais que incidiram sobre sistemas de processamento mais arcaicos, nos quais sucessivamente se resolveu a associação entre soluções possíveis para problemas reais, mutações não letais e cruzamentos.

Quanto ao segundo, alinha-se à discussão entre concepções corpo/ mente monistas e dualistas, onde se filia à tradição antimetafísica, particularmente ao empenho de superação do dualismo cartesiano, que, como destaca Antonio Damasio (1994a, 2003) já se fazia presente na obra de Espinosa, tendo se desenvolvido em sentido às perspectivas atuais em William James e na tradição empirista associacionista, mas que - em termos epistemológicos - encontrou na obra seminal Fenomenologia da Perсерção (Merleau-Ponty, 1962) grande desenvolvimento.

Entrementes, seguindo a trajetória histórica que se inicia com o pósestruturalismo, é interessante notar que, para além da superação de questões mais teóricas do que práticas, novos desenvolvimentos de oposição à "hegemonia do Cogito" produziram uma situação esdrúxula: a formação de uma tradição de críticas antirracionalistas, ancorando o prolegômeno de que a racionalidade não merece nenhum interesse, por ser um 'pré-juízo' de um sistema filosófico e não de uma condição ontológica - levando assim muitas pessoas a dedicarem suas vidas a criticar aquilo que, em sua essência, nunca tentaram entender.

Em oposição a esta tendência, a maioria dos autores que atuam no campo teórico de tomadas de decisão assume que a modelagem racional é uma das grandes conquistas no entendimento desta categoria de processos cognitivos, não por qualquer razão teórica, mas simplesmente porque a aplicação de hipóteses desta natureza conduz á confirmação de previsões em um sem fim de contextos (Johnson-Laird, 1988). 
Neste mesmo sentido, os críticos da análise racionalista, baseiam-se em relativizações de mesma monta: em muitas circunstâncias, despontam contradições entre previsão e fato que parecem não ser adequáveis a quaisquer torceduras do modelo, o que favorece a perspectiva de que modelos racionalistas são aplicáveis em algumas circunstâncias, mas não em todas. Como assinalou Daniel Kahneman:"o modelo do agente decisional racional foi nosso ponto de partida e a principal fonte de nossa (com Amos Tversky) hipótese de base" (Kahneman, 2003a, p. 1449), o que deve ser entendido simultaneamente a: "teorias do pensamento intuitivo não podem ascender à elegância e precisão dos modelos normativos formais de crenças e escolhas, mas isto é simplesmente um outro jeito de dizer que os modelos racionalistas são psicologicamente inconsistentes" (p. 1449). Do que seria oportuna a explicitação da ruptura em curso e, portanto, de que tipo de racionalidade está se falando.

\section{Utilidade Esperada: base da Moderna Teoria de Tomada de Decisões}

A Teoria dos Jogos, primeiramente formalizada por Von Neumann e Morgenstern (1944), trouxe o alento necessário para que os pesquisadores interessados em tomadas de decisão passassem a se mobilizar para entender esta dinâmica, tanto em nível individual quanto coletivo.

Por "jogos" entende-se uma relação sujeitos (ou um só sujeito), dotados da possibilidade de realizar determinadas ações (consideradas estratégias), as quais produzem consequências sobre os recursos que alocam.

Tradicionalmente, considera-se que os jogos podem ser cooperativos ou não cooperativos, tendo respectivamente soma não zero e soma zero. Jogos cooperativos são jogos em que os dois participantes tiram vantagem ou sofrem desvantagens de potenciais colaborações; diz-se que eles têm soma diferente de zero porque os ganhos ou prejuízos de todos juntos produzem um resultado diverso da soma dos recursos iniciais deles. Jogos não colaborativos são jogos em que as vantagens de um representam o prejuízo de outros; eles são considerados de soma zero porque a soma dos ganhos dos participantes equivale ao que todos juntos perderam. Em geral, pode-se dizer que as trocas materiais são predominantemente de soma zero, enquanto as relações sociais espontâneas e outras afins são de soma não zero.

Focando especialmente os jogos de dois jogadores com soma zero, Von Neumann e Morgenstern levaram a cabo uma inovadora sistematização lógica das trocas econômicas, inspirada nos objetivos e trocas de posições vantajosas/desvantajosas, a qual foi subsequentemente desenvolvida por vários pesquisadores, entre os quais John Nash, que amplificou a análise dos jogos não cooperativos, demonstrando a existência de pontos de 
equilíbrio, representativos das opções existentes no universo de cada um (Nash, 1951).

Muito pouco tempo após sua apresentação inicial, esta classe de análises matemáticas das trocas econômicas já havia se convertido em um dos mais influentes paradigmas em tomadas de decisão, o que se estende até hoje: muitas situações sociais complexas podem ser compreendidas à luz da teoria dos jogos, da mesma forma como determinados jogos artificiais alicerçam análises agudas das tomadas de decisões em séries curtas e extensas, como é o caso para o Dilema do Prisioneiro, que inspira análises das relações entre indivíduos e coletividade (Kollock, 1998), que pode se aplicar a nuances específicas como "a diferença entre relações interindividuais e intergrupais" (Insko et al., 1994), a modelos evolucionários de altruísmo recíproco entre estranhos (Killingback \& Doebeli, 2002).

Este dilema propõe a seguinte conjuntura: dois suspeitos de um crime são presos e separados; a cada um deles é oferecidos um jogo: se denunciar o outro e não for denunciado, será liberto, enquanto o outro cumprirá pena grave (dez anos); se ambos se denunciarem, cumprirão pena média (cinco anos); se ninguém se denunciar, cumprirão pena leve (seis meses). $O$ jogo pode ser jogado em rodadas nas quais entram em jogo as reações aos desenlaces anteriores.

O aspecto mais interessante do jogo é a sugestão de que, em determinadas circunstâncias, pode surgir uma contradição entre decidir pelo que parece mais vantajoso e decidir mais vantajosamente. Mais especificamente: existem contradições entre decisões egoístas, que podem ser frutíferas em curto prazo, mas que tem pouca chance de sê-lo no longo, e tendências crescentemente altruístas, que se farão vantajosas em série, conquanto haja reciprocidade, caracterizando nas palavras de Axelrod (1980): "a tensão entre racionalidade individual e racionalidade grupal" (p. 379). Formalmente, trata-se de um jogo de soma zero, cuja reiteração abre um flanco de tipo não zero.

E tal como sua análise destaca, muitas decisões que podem parecer irracionais à primeira vista mostram-se racionais sob análises apropriadas. Para que estas se revelem, a análise de tomadas de decisão entre seres humanos assume como máxima que nenhuma estratégia pode se mostrar por natureza menos ou mais atraente do que outras, a não ser que leve em consideração a estratégia do outro, ou ao menos que alguma assunção seja implicitamente assumida sobre o comportamento do outro jogador.

Só a título ilustrativo: no caso do dilema do prisioneiro, uma vez que este outro seja alguém assumidamente inteligente e voltado à maximização pessoal, a melhor estratégia consistirá em: 1. “Iniciar rodadas generosamente e então fazer exatamente aquilo que o outro jogador fez em seu movimento anterior" (Axelrod, 1980, p. 380). Esta estratégia foi desenvolvida por Rapoport e Chammah (1965). Uma vez que o outro seja incapaz de 
raciocínios de longo prazo (mantendo-se sempre egoísta), a melhor opção será iniciar as jogadas denunciando-lhe.

Em jogos deste tipo, assumimos que exista algo intrinsecamente reforçador em pauta, isto é,que os participantes emitam operantes em função de estímulos de alta valência (geralmente incondicionados). Não obstante, situações complexas frequentemente envolvem relações diferenciais com vários reforçadores, bem como problematizam a relação entre intensidade do reforço/investimento do sujeito. É sob este enfoque que destacamos outro desenvolvimento fundamental de Von Neumann e Morgestern, este especialmente importante para a análise de decisões individuais: o uso da noção de "utilidade", em uma lógica de "loterias" para a previsão e interpretação do comportamento decisional de sujeitos hipotéticos ou reais.

Utilidades são objetos do investimento dos participantes de um jogo. De acordo com a teoria, considerando que em uma sequência de escolhas um sujeito privilegie a opção por algo em detrimento de outra possibilidade, privilegie esta última em relação a uma terceira e assim por diante, poderemos inferir uma hierarquia utilitarista, através da qual números poderão ser atribuídos a cada uma das opções, quantificando o grau de preferência e, assim, de tomada de decisão, sob a máxima de que o jogador age teleologicamente.

Em face desta última, podemos ainda supor que a organização hierárquica das opções obedeça a princípios "racionais", isto é, alinhados com o termo geral, que é a maximização da utilidade. Racionalidade é definida de acordo com os seguintes axiomas: 1) Substituição (também chamado de "cancelamento"): se um jogador prefere " $A$ " em relação a " $B$ ", não pode preferir " $B$ " em relação a " $A$ ", uma vez que as outras condições do problema permaneçam constantes; 2 ) Invariância: descrições distintas de um mesmo problema, vistas pelo agente decisional como equivalentes, produzem as mesmas tendências decisionais (Tversky \& Kahneman, 1986); 3) Transitividade: se este agente prefere " $A$ " em relação a " $B$ " ${ }^{\text {" }} B$ " em relação a " $C$ ", deverá preferir " $A$ " em relação a " $C$ "; 4) Completude: dado um elemento " $C$ " e um jogador que prefere " $A$ " em relação a " $B$ ", este jogador saberá classificar " $C$ " como melhor que ambos, preferível a "B", ou inferior a ambos. ${ }^{2}$ Geralmente, Substituição e Transitividade são agregadas em um só princípio denominado Dominância, considerado a pedra de toque da teoria.

Loterias, por sua vez, são alternativas com consequências incertas e, portanto, riscos embutidos, os quais são objetiváveis, na medida em que são representados por probabilidades de ocorrência previamente delineadas, geralmente relativos a acontecimentos futuros (Mas-Colell, Whinston, \& Green, 1995). Nestes casos, diz-se que o jogador escolhe entre alternativas, de acordo com suas expectativas.

2 Frequentemente é mencionado o Princípio de Arquimedes, que não entra na definição de racionalidade, mas é necessário para alguns desenvolvimentos matemáticos: Para todo $p, q, r$ $\in P$, tal que $p>q>r$; existirá $a, b \in(0,1)$ tal que $a p+(1-a) r>q>b p+(1-b) r($ Buffet, 2004). 
Este braço fundamental da teoria retoma assim o conceito de Utilidade Esperada, cunhado por Daniel Benoulli no século XVIII (Kendall et al., 1961), revestindo-o das conjecturas delineadas à luz dos desenvolvimentos propiciados por Morgenstern-Von Neumann, que levam à construção de "funções lineares de valor utilitarista" (côncavas ou convexas ${ }^{3}$ ), simultaneamente sensíveis à atratividade da commodity, ao grau de incerteza e/ou expectativa de consegui-la e ao grau de aversão/propensão ao risco ${ }^{4}$. Estas funções são convencionalmente chamadas de funções Morgenstern-Von Neumann e vêm auxiliando a compreensão do comportamento complexo de jogadores sociais, ${ }^{5}$ dados seus investimentos em aplicações econômicas, consumo de bens não duráveis, relações familiares, escolhas profissionais, paliativos para retardar a morte etc.

Apenas a título ilustrativo, consideremos o seguinte exemplo: suponha que a utilidade de um agente seja expressa por $\sqrt{x}$. Se um jogo promete $\mathrm{R} \$ 4$ ou nada, o jogador terá utilidade igual a 2 ou zero respectivamente. Se as probabilidades forem de $50 \%$ para cada possibilidade, a Utilidade Esperada do agente será $1(0,5 \times 0+0,5 \times 2)$. Sabemos, por outro lado, que o valor esperado do jogo é $2(0,5 \times 0+0,5 \times 4)$. Se o agente possuir o valor esperado do jogo, sua utilidade será $\sqrt{2}$, que é superior a 1 . Ou seja, se o agente possuir exatamente o valor esperado do jogo em suas mãos, ele terá uma utilidade maior do que se ele participar do jogo. De onde decorre esta diferença? Decorre do risco que existe ao participar do jogo e que não ocorre quando o agente tem o valor esperado do jogo. Essa função utilidade expressa, portanto, as preferências de um agente que prefere o valor esperado do jogo a participar do próprio jogo e, assim, as preferências de um agente avesso ao risco.

Já se repetíssemos o exemplo anterior substituindo a função utilidade por $x^{2}$, chegaríamos exatamente ao resultado oposto, em que o agente iria preferir participar do jogo que ter o valor esperado do jogo garantido em suas mãos, o que o definiria como propenso ao risco. E, como é de se notar, teríamos um comportamento que a maioria das pessoas julgaria como "estranho". O que revela uma coisa importante sobre o comportamento da média da população mundial: quando se trata dos investimentos descritos acima (de aplicações econômicas a paliativos para retardar a morte),

3 É matematicamente demonstrável que funções utilidades côncavas representam preferências de indivíduos avessos ao risco, enquanto funções convexas representam preferências de indivíduos propensos ao risco. Nas primeiras, a utilidade do valor esperado, isto é, a utilidade do valor em média pago pelo jogo, é maior do que a utilidade esperada, ou seja, maior do que a utilidade de participar do jogo; nas segundas, ocorre o inverso.

4 Conforme a nomenclatura estabelecida por Knight (1921), incerteza se diferencia de risco, em função do nosso conhecimento das probabilidades. Como notou Green e colaboradores (MasColell et al., 1995), esta distinção desaparece na teoria de Von Neumann e Morgenstern.

5 Não podendo ser descartado que diferentes preferências são representadas por diferentes funções (Burton, 1977). 
as funções côncavas são mais comuns do que as convexas. Opostamente, quando se trata do comportamento de jogadores compulsivos (entre outros) este frequentemente expressa delineamento monotônico convexo ou quase convexo.

Um último aspecto a se notar é o fato de que Von Neumann foi um dos pioneiros da computação e que isto se reflete em parte nesta sua modelagem do comportamento decisional: por um lado, supõe-se que o comportamento decisional seja coerente ou algorítmico; por outro, o conceito de racionalidade agrega os gostos individuais e, portanto, um apelo idiossincrático, mediante o qual o valor absoluto dos objetos de desejo nunca poderia ser categoricamente problematizado por um agente externo, já que este não possui a força de influenciar sua avaliação.

Assim é que, apesar de sua simplicidade, os usos da teoria se tornaram amplos, o que contribuiu para que se confirmasse a previsão de (Copeland, 1945), de que a posterioridade provavelmente viria a considerar a obra seminal dos autores (Von Neumann \& Morgenstern, 1944) uma das contribuições científicas mais importantes da primeira metade do século XX'; fato este especialmente saliente dado que a modelagem desenvolvida à luz de conceitos racionais se converteu em verdadeira pedra de toque da racionalidade (Grafstein, 1995), definível desde então como "busca da maximização da utilidade esperada".

\section{A relativização da Utilidade Esperada}

Em 1953 a Teoria da Utilidade Esperada sofreu seu primeiro revés, do qual não sairia incólume. Através de um experimento simples, Maurice Allais (para um sumário das contribuições do autor, ver Allais, 1991) demonstrou que funções monotônicas poderiam não ser ideais para a caracterização do processo decisional, bem como que a axiomatizada transitividade entre utilidades (" $\mathrm{A}$ " > " $\mathrm{B}$ "> " $\mathrm{C}$ "logo " $\mathrm{A}$ " $>$ " $\mathrm{C}$ ") não seria sustentável em qualquer situação. Este ficou conhecido como 'paradoxo de Allais', ainda que não seja um paradoxo, já que o que ele contradiz não é uma assunção supostamente inviolável, mas uma teoria (Conlisk, 1989).

Para tanto, Allais usou a seguinte conjuntura: de início, os sujeitos deveriam escolher entre duas opções: (a) 100\% de chance de receber um milhão; (b) $10 \%$ de chance de receber cinco milhões, $89 \%$ de receber um milhão e $1 \%$ de não receber nada. Em uma segunda rodada, os sujeitos eram novamente apresentados a duas opções: (c) $11 \%$ de chance de receber um milhão e $89 \%$ de não receber nada e (d) $10 \%$ de receber cinco milhões e $90 \%$ de não receber nada. A grande maioria escolheu a primeira e a quarta opção.

Como é de se notar, o valor de "b" supera o de "a" em 39\%. Sob os paradigmas da Teoria da Utilidade Esperada, dizemos que esta opção (que produz uma função côncava) reflete aversão ao risco. Porém, "d" é menos 
arriscada do que "c", subvertendo o paradigma racionalista de manutenção coerente dos princípios decisionais (axioma da Invariância) ao longo de uma série.

Contrariamente à assunção de que a Teoria da Utilidade Esperada seria uma teoria fidedigna ao modo de ação do sujeito normal, uma teoria descritiva, o "paradoxo de Allais" inaugurou uma tradição de considerá-la uma teoria que, sob perspectiva hermenêutica, seria mais apropriadamente acolhida entre os modelos que versam sobre a maneira como as pessoas deveriam agir; uma teoria normativa portanto (Payne, Bettman, \& Johnson, 1993; Tversky \& Kahneman, 1986).

Neste sentido, vicejaram estudos relativos à circunscrição e sistematização destes comportamentos que rompem com as expectativas racionalistas, sob a ascendência de Kahneman e Tversky (Kahneman, 2002, 2003a, 2003b; Kahneman \& Tversky, 1979; Thaler, Tversky, Kahneman, \& Schwartz, 1997; Tversky \& Kahneman, 1974, 1981, 1986, 1991; Tversky, Slovic, \& Kahneman, 1990), os quais tiveram como estopim a descoberta do fenômeno denominado "negligência das taxas de base" (Kahneman \& Tversky, 1973), relativo à sistemática desconsideração dos efeitos de uma amostra sobre a probabilidade de um resultado.

O fenômeno da negligência de taxas de base se mostrou um dos mais significativos da literatura sobre desvios heurísticos na análise de critérios para tomadas de decisão, dando origem a um importante conceito sobre o funcionamento da mente humana:a chamada "lei dos pequenos números", isto é,"o exagero ao considerar a possibilidade que uma amostra pequena expresse o comportamento de uma população" (Rabin, 2002, p. 775).

Particularmente, quando as pessoas são impelidas a decidir se um elemento " $C$ " é mais apto a fazer parte de um grupo " $A$ " ou um grupo " $B$ ", tendem a desconsiderar a importância das relações $A /$ mostra e $B /$ mostra. Por exemplo: imagine um estabelecimento comercial diariamente visitado por $95 \%$ de mulheres e $5 \%$ de homens. Você é uma dessas pessoas, escolhe um produto e se dirige ao caixa; lá percebe que sua carteira fora roubada.É possível que você acredite que foi "um batedor de carteira" que te roubou. Ainda que a resposta 'uma batedora de carteira' se escore numa relação de dezenove para um, a qual, à luz da proporção mulheres/homens batedores de carteira da população como um todo, revele-se desfavorável para a assunção efetuada.

Um aspecto digno de nota é o de que a negligência sistemática das taxas de base não se opõe ao princípio de que a maior parte das decisões pessoais possa ser compreendida como investimentos para a maximização da utilidade esperada. O que ela contradiz é o princípio subjacente, de que as pessoas vão utilizar para tanto a probabilidade do evento-alvo ocorrer. Ou seja, a teoria contradiz a assunção excessivamente matemática sobre os parâmetros que as pessoas em geral se utilizam para fazer estimativas, sendo frequentemente compreendida como uma contradição à propriedade 
de simulações bayesianas ao raciocínio estatístico das pessoas em geral ${ }^{6}$ (Grether, 1980), especialmente saliente em situações de pressão temporal (Callen, 1991). De outro modo: a perspectiva não é de oposição irrestrita ao caráter descritivo da Teoria da Utilidade Esperada, mas à assunção de que os jogadores tenham a devida clareza e tempo para maximizar jogos cotidianos. Tanto no que tange ao experimento de Allais (Conlisk, 1989), quanto à negligência das taxas de base (Tilmann Betsch, 1998), esta assunção está de acordo com experimentos que demonstram que o efeito"paradoxal" varia em função da maneira como as hipóteses são apresentadas.

Porém vários efeitos de alterações de preferências, contraditórios aos fundamentos teóricos da Teoria da Utilidade Esperada (especialmente em relação ao Axioma do Cancelamento) foram demonstrados, formando uma classe de efeitos chamados 'Efeitos do enquadramento (Framing Effects) (Robert, 1993; Tversky \& Kahneman, 1981; Tversky \& Kahneman, 1986). Por exemplo, dado um problema mediante o qual um sujeito qualquer deve decidir se sacrifica ou não um número pequeno de pessoas para salvar uma população maior; se este for apresentado de duas maneiras distintas, uma frisando os que se salvarão e outra frisando os que morrerão, é possível que o sujeito escolha diversamente.

Expandindo esta linha de pesquisas sobre heurísticas e Desvios, foram apontados três tipos fundamentais de desvios estatísticos:ancoragem, disponibilidade e representatividade (Tversky \& Kahneman, 1974).

A regra dos pequenos números é a essência dos processos heurísticos caracterizados por representatividade e disponibilidade. Já por ancoragem (especificamente: ancoragem e ajuste), entende-se a sistematização de observações relativas ao fato de que as pessoas tendem a estabelecer valores probabilísticos para eventos incertos de maneira consideravelmente contingente (ancoragem), passando subsequentemente para uma etapa de correção do mesmo, em função de outros valores enunciados ou memorizados.

Mesmo valores irrelevantes podem ser levados em conta, tal como se a tendência geral dos jogadores fosse a diminuição da dissonância entre estes valores. Por exemplo, suponha que o último dígito do seu CPF seja 9 e que o correlato de seu colega seja 3 e que eu peça para vocês memorizarem este número e, em seguida, para avaliarem em uma escala de 0-10 quanto valeria um determinado produto que nenhum de vocês têm qualquer noção objetiva de seu preço de mercado. É possível que você dê uma nota mais alta do que ele. Ou melhor: considerando cem mil pessoas (evitando Desvios de pequenos números), é provável que os que leram 9 atribuam notas mais altas de maneira estatisticamente significante.

Uma outra forma mais aplicável à vida real de se aproximar do fenômeno é através de jogos de soma zero, em que um é o vendedor e o

6 Há correlações entre estimativas bayesianas e pensamento utilitarista, mas as duas coisas não podem ser confundidas. 
outro um comprador final. Caso o preço inicial do produto seja mais alto, é provável que a barganha apresente também valor mais alto do que se o valor inicial fosse menor.

O mesmo se aplica à lógica que guia a quantidade de produtos que uma pessoa se mostra disposta a comprar em uma situação convencional de varejo. Em um artigo voltado ao desenvolvimento de estratégias para fazer o consumidor comprar mais produtos de uma só marca, ${ }^{7}$ Wansink et al. (1998) demonstraram o caráter experimentalmente válido da seguinte estratégia: fazer promoções de produtos embalados em grandes quantidades, para ancorar o conceito "grande quantidade", aumentando assim a venda dos outros produtos, vendidos em preço cheio.

Aproveitando a oportunidade, ressaltamos um uso inédito do conceito para a interpretação de um fenômeno aparentemente obtuso: a tendência aparentemente injustificada de alguns supermercados anunciarem como ofertas produtos cujos preços estão cheios (ou mais do que isso), ainda que se tratem de produtos de amplo consumo e valores relativamente constantes entre os estabelecimentos e, assim, conhecidos dos consumidores (exemplo: pães, café, carne), explica-se posto que estes preços sabidamente distorcidos servem para ancorar a impressão de que "os preços subiram" e, por extensão, de que os outros produtos estão baratos.

Por fim, também chamamos a atenção para o fato de que uma série ampla de falsos silogismos (definidos por Charles Sanders Pierce como abdução ou "associação objetiva entre fenômenos independentes, com aspectos descritivos semelhantes") podem ser compreendidos como desvios heurísticos de tipos diversos e, porventura, de tipos mistos. Por exemplo:é comum se considerar que enxadristas são pessoas inteligentes, entre outras coisas, por também lidarem com outras atividades que a maioria das pessoas relaciona com alta inteligência, como a matemática; assim é que se aceita tacitamente que o xadrez exija inteligência (o que está certo) e então que "a inteligência seja um critério diferencial entre os bons enxadristas". Contrariamente, foi demonstrado (Bilalic, McLeod, \& Gobet, 2007) que, entre jogadores de elite, medidas experimentais de inteligência média são inversamente correlacionadas à desenvoltura no jogo e diretamente correlacionada ao quanto se pratica.

Mais do que apontamentos específicos, a percepção da imanência dos desvios heurísticos abriu uma senda para descobertas inumeráveis. Todavia, foi saindo do domínio estimativo e entrando na dinâmica dos processos de escolha que o revés iniciado por Allais deu forma a uma teoria: a chamada teoria prospectiva, cujo surgimento se deu a demonstração de inconsistências na função monotônica Morgenstern-Von Neumann (Kahneman, 2003b; Kahneman \& Tversky, 1979).

7 "Nossa intenção é iluminar a psicologia que governa o modo como as pessoas fazem decisões de compras quantitativas e sugerir como marqueteiros podem influenciar esta decisão" (Wansink et al., 1998, p. 71) 
Naquela que talvez seja a mais célebre demonstração de sua parceria, os novos desbravadores (Kahneman \& Tversky, 1979) demonstraram que as pessoas se tornam mais avessas ao risco em função de uma sequência de ganhos e mais afeitas a assumi-los quando estão perdendo, ou seja, revelam uma frequente "reversão de preferência" (Tversky \& Kahneman, 1982; Tversky et al., 1990), o que preconizou o abandono do modelo monotônico, bem como uma reviravolta na própria atribuição de valores, que passaram das contingências finalistas aos ganhos e perdas do processo.

Paralelamente, foram alinhavadas evidências de que as pessoas atribuem mais valor a resultados certos em relação a resultados prováveis e que tendem a atribuir valores não lineares em função da improbabilidade de uma ocorrência (Kahneman, 2003a, 2003b).

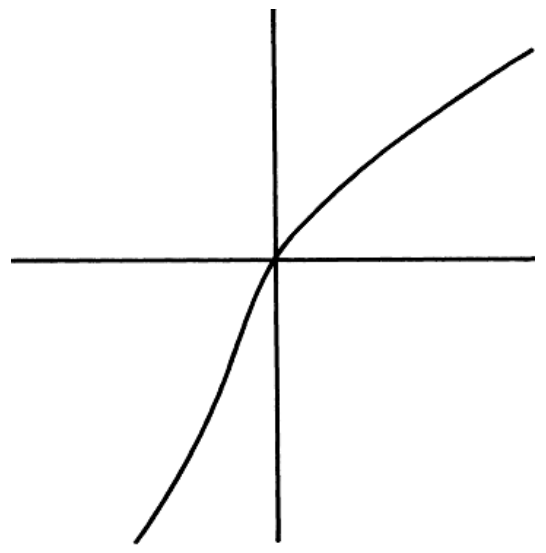

Figura 1. (perdas -- ganhos/ $\mathrm{F}(\mathrm{x})=$ valor)

Função de valor típica (Tversky \& Kahneman, 1986, p. 259)

Mas, se assim é,como explicar a extensão do pensamento heurístico? Seria este tão somente um reflexo da irracionalidade humana, de sua incapacidade de maximizar seus recursos, de uma forma de raciocinar que, tal como assinalado por Oppenheimer e colaboradores (Alter, Oppenheimer, Epley, \& Eyre, 2007) diminui em função de reflexões metacognitivas? Ou, opostamente, haveria outras tantas sutilezas sob o manto da desrazão?

Na realidade, os desvios heurísticos são parte de uma categoria de processos cognitivos cujo balanço de custos/benefícios (ainda que impossível de mediação objetiva) parece resultar francamente positivo, representando uma maneira de maximizar o aproveitamento dos recursos cognitivos limitados que temos a nossa disposição. Por exemplo: consideremos um jogador de xadrez profissional em um campeonato, frente a um sem fim de possibilidades de jogo; será que ele seria capaz de computar todas as jogadas possíveis, avaliando-as e escalonando as mais vantajosas, antes 
que soe o gongo, encerrando sua rodada? Ou seria mais vantajoso utilizar um processo heurístico, que de antemão excluísse a imensa maioria das jogadas de consideração analítica?

No âmbito da aplicação da Teoria dos Jogos para interpretação e maximização das estratégias de enxadristas, destaca-se que a "acurácia avaliativa decresce em função da profundidade da busca pelo resultado perfeito" (Sadikov, Bratko, \& Kononenko, 2005), procedimento por sua vez denominado "minimax patológico" (Lustrek, Gams, \& Bratko, 2006; Sadikov et al., 2005).

Mais amplamente, todo expert é um pensador heurístico.O mesmo se dá na área da saúde (Christensen \& Hewitt-Taylor, 2006; Effken, 2001; Leskowitz, 2002), entre executivos (Hung, 2003; Ritchie, Kolodinsky, \& Eastwood, 2007), artistas (Horter, 1934; Perkins, 1977) e possivelmente em qualquer outra área.

E isto justamente produz uma dicotomia utilitarista no seio do pensamento heurístico, a qual Baylor (2001) propõe solucionar com um modelo em forma de $U$ para dois tipos de intuição heurística: madura e imatura.

\section{Da heurística intuitiva à intuição}

Entre os vários aspectos que diferenciam "heurísticas decisionais" de "intuição" é o fato de que "heurística" significa "modo simplificado de pensar", bastando-se pela circunscrição de fenômenos objetivamente irracionais, tais como a negligência das taxas de base, enquanto "intuição" aponta para uma perspectiva de sistema, o que por sua vez pode incorporar expressões difíceis de circunscrição objetiva. De outro modo:a etiologia de uma heurística pode ser estabelecida em função de condicionamentos, enquanto a da intuição pode ser mais ampla, ainda que todos estes conceitos se remetam à noção de processos não redutíveis à modelagem racional e a algum grau de inconsciência.

Tal como destacam Pretz e Totz (2007), a história recente da intuição, abrange duas categorias teóricas, holística e heurística. A primeira enfocando a importância das experiências emocionais e/ou subjetivas e a segunda enfocando os dispositivos para pensar. Uma derivação do modelo de heurística e Desvios de Kahneman e Tversky em forma de sistema possui posição de destaque, sob esta última acepção de intuição.

Os criadores da teoria prospectiva propuseram a existência de dois sistemas de pensamento, ativos nas tomadas de decisão:"sistema 1, rápido, automático, não esforçoso, associativo e de difícil controle e modificação...

É comum se dizer que se tratam de "corta-caminhos cognitivos para reduzir problemas complexos em problemas mais simples e, por extensão, julgáveis" (Shiloh, Salton, \& Sharabi, 2002). Isto está sutilmente errado, conquanto não há necessariamente um "para" ou investimento intencional. 
sistema 2, mais lento, serialista, esforçoso, deliberadamente controlado, flexível e potencialmente controlável por regras" (Kahneman, 2002, p. 2).

Segundo eles, o sistema 1 produz "impressões sobre os objetos do pensamento e percepção",enquanto o sistema 2 produz juízos (p. 2), sendo o primeiro "intuitivo" e o segundo "deliberativo" e de tendência mais explicitamente utilitarista; a primeira relação com os dados de um problema é estabelecida pelo sistema 1 e, em segunda instância, submetida aos escrutínios do sistema 2.

Na nossa visão, esta formulação permitiu uma inovadora (e nunca antes enunciada) contraposição ao próprio paradigma intencional/teleológico, propiciando uma visão parcimoniosa da dicotomia determinismo/ livre arbítrio: as pessoas têm a possibilidade de exercer o livre arbítrio e o fazem quando definem para si uma utilidade e investem recursos para atingi-la, apenas não podemos ingenuamente considerar que esta condição seja imanente, nem que o processo de tomada de decisão reflita, indiferenciadamente, pulsões teleológicas.

Assim também, a relação em parte sinérgica e em parte opositiva (tal como assinalado na introdução) destas novas teorias com os modelos racionalistas derivados do utilitarismo ganha consistência programática. O sistema 2 nem sempre é ativado (por exemplo: uma decisão sob forte tensão temporal não o permite) e, quando o é, recebe inputs já assimilados pelo sistema 1, ou seja, não opera com "informações idôneas", do que se explica porque uma situação confortável de ganhos diminui a tendência ao risco, enquanto a situação inversa aumenta: estas avaliações são feitas em função da experiência de se ganhar e de se perder, não da variável utilidade esperada, por excelência finalista.

Mais amplamente: as avaliações se dão em função de tendências (retorno ao paradigma ordinal, abandono do cardial) e estas tendências se escoram na maneira como as situações de jogo despertam, pontualmente, modos de ação subsequentes e isto é parte de uma condição ampla do modo como a mente humana funciona, denominada intuição.

Porém o modelo não é livre de críticas e complementos, entre os quais merece atenção o modelo de Epstein (2008), denominado CEST (cognitive-experiential self-theory), o qual, em primeira instância faz coro em relação à possibilidade de se dividir o sistema cognitivo em dois grandes módulos, sendo um racional e outro intuitivo, ${ }^{9}$ enquanto em segunda instância sugere que os dois subsistemas operariam em paralelo e possuiriam interatividade bidirecional. "O sistema experimental automaticamente estabelece associações através de condicionamento clássico, condicionamento instrumental e aprendizado observacional, em todas as formas envolvendo relações com estímulos elou respostas com resultados. O sistema racional é um sistema lógico que opera de maneira consciente, verbal,

9 Um coro que remonta à antiga dicotomia dualista faculdades sensuais/faculdades noéticas, inspirado por Locke. 
abstrata, analítica, livre de afetos e com alta demanda cognitiva" (Epstein, 2008, p. 25).

Esta oposição entre encadeamento serial (Kahneman eTversky) e paralelo (Epstein) do processamento não racional envolve mais do que simplesmente um detalhe conceitual: retomando a assertiva de que "intuição" é um conceito para se falar de etiologia (que de resto é francamente vazio), denota-se que a oposição aponta para etiologias divergentes, bem como para diferentes maneiras de se pensar a atuação de sistemas automáticos ou "inconscientes".

Ao passo que podemos fazer uma analogia entre a concepção de inconsciente de Kahneman e Tversky e a máxima fenomenológica de que "o inconsciente não está atrás, mas é como um véu que encobre a consciência" (Merleau-Ponty, 1962), podemos associar a concepção de Epstein àquela lâmina inamovível, que ocupa o platô inferior $\left(3^{\circ}\right)$ do modelo óptico de Freud (1900/1953). ${ }^{10}$ Em outras palavras, para Kahneman e Tversky, o inconsciente é aquele primeiro estágio que se atravessa e que indelevelmente deixa as suas marcas; para Epstein, ele é aquele nunca de todo atravessado.

Esta diferença se torna clara ao analisarmos um problema específico, como o de que uma série de ganhos torna os jogadores mais conservadores e uma série de derrotas os tornam mais ousados (reversão de preferências). De acordo com Kahneman e Tversky (1982), isto ocorre porque as pessoas têm uma compreensão distorcida de fenômenos probabilísticos em função da tendência de associar dogmaticamente o comportamento de mostras pequenas ao de mostras grandes (heurística representacional, especificamente, lei dos pequenos números). De acordo com o modelo de Epstein, podemos supor que elas seriam guiadas por sua experiência: quando se está ganhando, o prazer (reforço) aumenta, tornando crescente a ameaça de perda; quando se está perdendo, o desprazer (punição) aparece como algo a ser evitado, do que surgem investimentos irracionais para tanto.

Apesar da elegância do modelo de Kahneman e Tversky destaca-se o fato de que existem situações em que a irracionalidade de expressão fenomenológica "intuitiva" se faz inamovível. É justamente isto o que ocorre nas mesas de bingo, no âmbito das intuições premonitórias entre outras de cunho negativo e algumas de cunho positivo.

A existência de um sistema em atividade perene em relação ao qual o processamento informacional retroage é clarividente e foi demonstrada por experimentos que denunciaram flagrante conflito entre "o coração e a cabeça" (Pacini \& Epstein, 1999). Em um experimento clássico, Epstein e colaboradores (Denes-Raj \& Epstein, 1994) ofereceram a seus sujeitos 1 dólar por cada bala vermelha que conseguissem tirar aleatoriamente, de um entre dois potes de balas coloridas, os quais continham, respectiva-

10 Como salientou Epstein (Epstein, 1994), a CEST (sua teoria) procura integrar o inconsciente cognitivo e psicanalítico. 
mente, maior número de balas premiadas e maior proporção destas. Os sujeitos exibiram predileção pelo primeiro pote, ao mesmo tempo em que relataram que sabiam que teriam mais chances no outro. Acreditavam que tirariam a sorte no primeiro. Em relação ao modelo sistema1/sistema 2, podemos resumir dizendo que "toda forma de intuição que não se abre a um prolongamento racional não é bem explicada desta maneira". O que por sua vez incentivou a elaboração de uma escala de estilos de pensamento - racional versus experimental, denominada REI (Rational Experintial Inventory) (Epstein, Pacini, Denes-Raj, \& Heier, 1996; Pacini \& Epstein, 1999).

Paralelamente, os fatos apontados por Epstein e colaboradores nos remetem à questão levantada na introdução:"o que é uma decisão"? Retomemos o que dissemos então:"a tomada de uma decisão expressa o investimento sobre uma aparição mental, tradicionalmente representativa de algo que existe no mundo, em detrimento de uma ou mais opções".

Considerando este sentido clarividente, perguntamo-nos: será que o modelo de Epstein pode ser considerado um modelo sobre tomadas de decisão? Mais amplamente, será que, no que tange à vida mental, faz qualquer sentido se modelar o funcionamento de operações discretas em função de parâmetros constantes? Provavelmente não. E é isto o que torna o modelo tão "holístico" quanto "experimental".

No que tange ao problema específico que enseja o campo das modelagens (ou teorias) de tomadas de decisão, a perspectiva de Epstein se conecta à do sujeito que diz que "a moça decidiu não saltar de paraquedas porque seu superego não a permitiu", ou que "ela decidiu saltar ao vencer estas barreiras". O problema com estas asserções é simplesmente o de que o superego já existia antes da decisão, bem como permanecerá existindo após, o que torna a asserção não informativa sobre a decisão em si, ainda que a torne informativa sobre o conceito de superego. ${ }^{11}$ É possível que o paralelismo de Epstein represente uma evolução da dicotomia sistema 1/ sistema 2 de Kahneman e Tversky; apenas não podemos deixar de ter em vista que a pedra de toque do trabalho deles é representada pelos padrões observados ao longo das escolhas das pessoas.

Mas, afinal, existiria alguma maneira de se estar simultaneamente voltado às decisões em seu caráter pontual e ao caráter holístico/fenomenológico da intuição? Respondendo a esta questão é que introduzimos o último modelo sobre o qual nos estenderemos: a hipótese do marcador somático (Bechara, 2004; Bechara \& Damasio, 2002, 2005; Bechara, Damasio, Tranel, \& Damasio, 2005; Damasio, 1994b).

Utilizando o jogo de apostas lowa Gambling Task, o qual envolve escolhas entre montes de baralho com índices de risco diversos, os autores

11 Existe um outro problema, que é o de saber se este tipo de abordagem depende da assunção de uma "cisão no âmago do ser", através do qual, partes extrapartes, possamos falar de uma parte de si atuando objetivamente contra outras. Mas isto foge dos nossos interesses atuais. 
mostraram que as pessoas exibem respostas antecipatórias de condução cutânea (SCR), típicas de estresse, quando tendem a jogadas cujo custo/ benefício de longo prazo é desfavorável, o que as faz abandonar esta opção ao longo de uma série (Bechara et al., 2005). E que isto ocorre à revelia da consciência. ${ }^{12}$

Para chegar a esta conclusão, os autores partiram da premissa de que a porção simpática do sistema autonômico está filogeneticamente relacionada a instintos de "correr e fugir", exibindo propriedades sinalizadoras pré-declarativas e pré-conscientes, fornecendo substrato à circunscrição de uma das expressões holísticas da intuição. Em seguida, mostraram que estas respostas são expressas quando ocorrem perdas no jogo e, por fim, que deste momento em diante, elas passam a se expressar quando os sujeitos saudáveis exibem a intenção de retirar cartas dos baralhos associados às perdas mais enfáticas. Lesões cerebrais, ${ }^{13}$ correlacionadas a maus desempenhos no jogo, foram associadas à baixa expressão do marcador somático.

Há poucas dúvidas de que o modelo seja representativo de uma nova etapa no processo de entendimento dos processos decisionais não racionais. Não obstante, é de se considerar que serve melhor à função de explicar porque as pessoas não fazem certas coisas do que porque o fazem.

Mais especificamente: consideremos o fato de que as pessoas preterem jogos mais arriscados por mais conservadores ao longo de uma série significativa, o que se correlaciona a respostas autonômicas simpáticas de alta ativação, fruto de condicionamento clássico estabelecido por meio de perdas catastróficas anteriores; disto se destaca que a não escolha de " $X$ " produz uma inclinação à $Y$, o que em situações multifatoriais é francamente insuficiente para se determinar uma dinâmica de escolhas multifatorial.

12 Este aspecto é enfatizado por Damasio, Bechara e colaboradores (Bechara et al., 2005); estes aplicaram um teste de memória de curto prazo junto ao IGT e perceberam que o desempenho não foi afetado (Turnbull, Evans, Bunce, \& Carzolio, 2005), o que indica rota alternativa à simbólico-declarativa. Por sua vez, (Maia \& McClelland, 2004,2005) aplicaram o teste perguntando aos sujeitos sobre suas percepções e assim concluíram que o efeito intuitivo não é inconsciente. Bechara e colaboradores (Bechara et al., 2005) contra-argumentaram que Maia e McClelland não interpretaram corretamente suas colocações, bem como apresentaram uma versão mais branda de efeito inconsciente. Nossos estudos, preliminares e não controlados, baseados em GSR/EMG/EEG, em um aparelho J\&J de 6-canais com interface humana pelo BioExplorer 1.3.0.270 e uma versão adaptada do teste, (que está disponível em: http:// users.fmg.uva.nl/rgrasman/experiment/lowaGamblingTask_en.html ) com tradução nossa, sugeriram que o efeito não é de fato inconsciente em estrito senso. Porém mais estudos são necessários, como apontaram (Sanfey \& Cohen, 2004).

13 Principalmente lesões na porção ventromedial do córtex pré-frontal (VMPFC) (Bechara \& Damasio, 2002). Edmund Rolls, por sua vez, destacou o papel da porção orbito-frontal para a execução da mesma tarefa (Kringelbach \& Rolls, 2004; O'Doherty, Kringelbach, Rolls, Hornak, \& Andrews, 2000; Rolls, 2004, 2006). Outros autores destacaram outras. 
Por outro lado, considerando que:"marcadores somáticos podem ser tanto positivos quanto negativos e, em situação de conflito e incerteza, ajudam a reforçar ou endossar opções de ação" (Damasio, Bechara, \& Damasio, 2002, p. 1104), a limitação é contornada ao custo de um esvaziamento do próprio poder etiológico, pois o caráter primordial de um processo informacional -a diferença - é abolida.

Porém a questão não versa sobre a melhor "estratégia retórica", mas sobre o que mostram os dados. Ainda que não existam estudos publicados focando diretamente esta questão, é mister considerar que as respostas de ataque e fuga são, por excelência, as mais intensas, já que, de outro modo, o organismo não sobreviveria para expressar as outras.

É justamente neste sentido que enfocamos a sua limitação, da qual emerge seu poder de fogo: o modelo se abre à possibilidade de se traçar curvas de atividade autonômica, a partir das quais poderão ser propostas estatísticas consistentes para a probabilidade de um sujeito experimentar uma experiência intuitiva de cunho holístico e de qualidade aversiva, mas não permite uma teoria geral da intuição holística.

\section{Considerações Finais}

Existem muitas metodologias e estudos específicos importantes, sobre os quais não pudemos nos verter na presente ocasião. Um deles versa sobre as estratégias que os não especialistas mais utilizam para fazer inferências em problemas decisionais com grande número de variáveis, que podem representar itens em alternativas, ou número de alternativas.

Em um clássico deste subdomínio da "heurística e desvios", em que Payne et al. (1993) se propuseram a alinhavar aquelas que Ihes parecem ser as principais estratégias, revelou-se que quando as opções todas evidenciam balanços favoráveis, mas ao mesmo tempo exibem variáveis internas de valência variável (por exemplo: ir para a praia, pegar um pouco de trânsito, ficar em um bom hotel/ir para a montanha, sem trânsito, ficar em um hotel ruim), as pessoas tendem a considerar comparativamente a pior variável de cada hipótese em estado de equilíbrio, eliminando aquela que exibir a variável de maior valência negativa.

Ou seja, a comparação das variáveis positivas é relegada a segundo plano, as hipóteses não são comparadas em seus valores absolutos e, com isto, toda a objetividade inferencial se torna enviesada.

Nós não nos estendemos sobre este tipo de conjectura,já que centramos nosso foco em jogos de uma ou duas opções, para assim evidenciar os elos mais significativos que norteiam as principais teorias. Contrariamente, uma das principais variáveis em jogo no estudo de Payne e al. (1993) é a capacidade de armazenamento da memória de trabalho.

Mas mesmo neste sentido restrito, como é de se perceber, existe uma já longa trilha a se distender desde o utilitarismo algorítmico da primeira 
metade do século XX à incorporação do sem número de efeitos da condição humana, não só em sua limitação computacional, mas em seus determinantes encarnados, em relação aos quais o uso do designativo "heurístico" sugere "work in progress", contínua agregação de novos achados.

"Holístico" tampouco aponta para qualquer tipo de holismo doutrinário, místico, mas simplesmente às extensões da mente que ultrapassam os limites epistemológicos de um sistema físico-simbólico, guiado por uma sintaxe implementacional que arquiteta operações discretas, obtendo destas um output cujo grau de correção é determinado pela associação objetiva entre princípios inferenciais sobre o espaço amostral e transformacionais sobre os paradigmas axiomatizados, cedendo aos efeitos quantitativos dos mecanismos pré-declarativos que imbuem saliência e valência (eventualmente como um sistema fuzzy) aos representantes de um esquema decisional.

É a esta associação heurístico-holística que se denomina "intuição decisional" (Pretz \& Totz, 2007) e é por força de sua multiplicidade e relação com a integração de sistemas de processamento informacional de tratamento diverso que seu entendimento permanece tão tímido.

Reconhecidamente, a intuição é um conceito de difícil definição (Anderson, 1926; Brown, 1914; Burtt, 1953; Newton, 1941), o qual foi fundamental para Kant (pura aparição), Schopenhauer (intuição volitiva), Bergson (relação imediata com o que se mostra), Moore (intuição ética), Fry (estética), Keynes (probabilística (Lang, 1964)), entre outros mestres.

Como assinalaram Yoeli, Manor e Gill (1989), programar a intuição computacionalmente é o maior desafio na emulação a inteligência humana em uma máquina. Neste sentido, o trabalho de Damasio, Bechara e colaboradores iniciou um processo de preenchimento desta lacuna "não amodal", mas dependente da corporeidade.

Ao mesmo tempo, criou-se com isto (especificamente: com o desenho dos circuitos morfofuncionais subjacentes, denominados "if' e "as if") potencial para que surjam modelos estatísticos que associem respostas eletrofisiológicas, relativas à probabilidade de um sujeito passar em algum momento a experimentar o que se denomina intuição holística, assunto sobre o qual estamos ativamente empenhados, sobretudo através de tentativas de encontrar correlatos das escolhas positivas e diferenciais eletrofisiológicos (EEG, EMG GSR) correlacionáveis a desempenhos em testes de intuição e a um teste computacional de desenvolvimento ad hoc (em parceria com Adrian Van Deusen).

Porém, grande parte ainda está por vir. Especificamente, é fundamental para a modelagem das intuições "em sentido a" que se desenvolvam mais protocolos "as if", em que o foco seja menos localizacionista e, tanto mais, centrado em mapeamentos de conectividade. 


\section{Reason and unreason in decision making}

Abstract: The current state of Decision Making Theories reflects the intersection of three major theoretical approaches: Expected Utility, Heuristics and Bias and Holistic Intuition. The relations between these approaches are not immediately evident nor even established in the literature, as some of these tendencies are quite new. My objective is to help to fill this gap by offering a general overview of the field, with particular attention to the epistemological conditions to which each new development responded and to the limitations of those responses. This overview will enable the reader to comprehend the conceptual foundations of intuitive decision making and to establish a critical position with respect to this emerging concept.

Keywords: Decision Making. Expected Utility. Heuristics and Bias. Holistic Intuition.

\section{Raison et déraison dans les prises de décision}

Résumé:Lechamps desThéories dePrises de Décision seprésente actuellement comme l'intersection de trois développements théoriques principaux: Utilité Attendue, Heuristique et Déviation et Intuition Holistique. Les relations entre eux ne sont pas claires et ne sont même pas établies dans la literature, surtout parce que quelques-unes de ces tendances sont très récentes. Mon objectif ici est de combler cette lacune, en offrant une vision générale du champs particulièrement sensible aux demandes epistémiologiques auxquelles chaque nouveau développement a répondu, et aux limitations de ces réponses. L'intéret particulier de ce travail est le fait qu'il permettra au lecteur de comprendre le nouveau concept d'institution décisionnelle qui apparait et de se positionner de façon critique en relation à celui-ci.

Mots clés: Prises de Decision. Utilité Attende. Heuristique et Deviation. Intuition Holistique.

\section{Razón y desrazón en las tomas de decisión}

Resumen: Tal como se presenta en la actualidad, el campo de Teorías de Tomas de Decisión refleja la intersección de tres enfoques teóricos principales: Utilidad Esperada, Heurística y Desvíos, e Intuición Holística. Las relaciones entre esos tres campos no son clarividentes, ni están establecidas en la literatura sobre el tema, especialmente porque algunas tendencias en juego son muy recientes. Mi objetivo es contribuir para suplir esa falta, ofreciendo una visión general del campo de trabajo, prestando especial atención a las condiciones epistemológicas, a las que cada nuevo desarrollo ha respondido hasta este momento, como así también a las limitaciones de esas respuestas. Entendemos que así ayudaremos al lector a comprender los fundamentos del nuevo concepto de intuición, en el proceso de toma de decisiones, y a posicionarse de manera crítica, respecto del mismo. 
Palabras clave: Tomas de Decisión. Utilidad Esperada. Heurística y Desvios. Intuición Holística.

\section{Referências}

Allais, M. (1991). An outline of my main contributions to economic Science. The Theory and Decision, 30(1), 1-26.

Alter, A. L., Oppenheimer, D. M., Epley, N., \& Eyre, R. N. (2007). Overcoming intuition: Metacognitive difficulty activates analytic reasoning. Journal of Experimental Psychology: General, 136(4), 569-576.

Anderson, F. (1926). Intuition. The Journal of Philosophy, 23(14), 365-377.

Axelrod, R. (1980). More effective choice in the prisoner's dilemma. The Journal of Conflict Resolution, 24(3), 379-403.

Baylor, A. L. (2001). A U-shaped model for the development of intuition by level of expertise. New Ideas in Psychology, 19(3), 237-244.

Bechara, A. (2004). The role of emotion in decision-making: Evidence from neurological patients with orbitofrontal damage. Brain and Cognition, 55(1), 30-40.

Bechara, A., \& Damasio, H. (2002). Decision-making and addiction (part I): Impaired activation of somatic states in substance dependent individuals when pondering decisions with negative future consequences. Neuropsychologia, 40(10), 1675-1689.

Bechara, A., \& Damasio, A. R. (2005). The somatic marker hypothesis: A neural theory of economic decision. Games and Economic Behavior, 52(2), 336-372.

Bechara, A., Damasio, H., Tranel, D., \& Damasio, A. R. (2005). The lowa Gambling Task and the somatic marker hypothesis: Some questions and answers. Trends in Cognitive Sciences, 9(4), 159-162.

Bilalic, M., McLeod, P., \& Gobet, F. (2007). Does chess need intelligence? A study with young chess players. Intelligence, 35(5), 457-470.

Brown, A. B. (1914). Intuition. International Journal of Ethics, 24(3), 282-293.

Buffet, S. (2004). Monte Carlo Algorithms for Expected Utility: Estimation in dynamic purchasing. Fredericton, NB: University of New Brunswick. 
Burton, A. W. (1977). Comparing utility functions in efficiency terms or, what kind of utility functions do we want? The American Economic Review, 67(5), 991-995.

Burtt, E. A. (1953). Intuition in eastern and western philosophy. Philosophy East and West, 2(4), 283-291.

Callen, C. R. (1991). cognitive science, bayesian norms and rules of evidence. Journal of the Royal Statistical Society. Series A (Statistics in Society), 154(1), 129-130.

Christensen, M., \& Hewitt-Taylor, J. (2006). Defining the expert ICU nurse. Intensive and Critical Care Nursing, 22(5), 301-307.

Conlisk, J. (1989). Three variants on the Allais example. The American Economic Review, 79(3), 392-407.

Copeland, A. H. (1945). Review: John von Neumann and Oskar Morgenstern, theory of games and economic behavior. Bulletin of the American Mathematics Society, 7, 498-504.

Damasio, A. (1994a). Descartes error. New York: J. P. Putnam's Son.

Damasio, A. (1994b). Descartes' error: Emotion, reason, and the human brain. New York: Avon.

Damasio, A. (2003). Loking for Spinoza: Joy, sorrow, and the feeling brain. London: Harvest Book.

Damasio, H., Bechara, A., \& Damasio, A. R. (2002). Reply to 'do somatic markers mediate decisions on the gambling task?' Nat Neurosci, 5(11), 1104-1104.

Denes-Raj, V., \& Epstein, S. (1994). Conflict between intuitive and rational processing: When people behave against their better judgment. Journal of Personality and Social Psychology, 66(5), 819-829.

Effken, J. A. (2001). Informational basis for expert intuition. Journal of Advanced Nursing, 34, 246-255.

Epstein, S. (2008). Intuition from the perspective of cognitive-experiential self-theory. In H. Plessner \& C. B. Plessner (Eds.), Intuition in judgment and decision making (pp. 23-39). New York: Lawrence Elrbaum.

Epstein, S. (1994). Integration of the cognitive and the psychodynamic unconscious. American Psychologist, 49(8), 709-724. 
Epstein, S., Pacini, R., Denes-Raj, V., \& Heier, H. (1996). Individual differences in intuitive-experiential and analytical-rational thinking styles. Journal of Personality and Social Psychology, 71(2), 390-405.

Freud, S. (1953). The interpretation of dreams (Vol. 4). London: Hogarth Press. (Trabalho original publicado em 1900)

Grafstein, R. (1995). Rationality as conditional expected utility maximization. Political Psychology, 16(1), 63-80.

Grether, D. M. (1980). Bayes rule as a descriptive model: The representativeness heuristic. The Quarterly Journal of Economics, 95(3), 537-557.

Horter, H. L. (1934). Intuition in modern painting. Bulletin of the Pennsylvania Museum, 29(162), 76-79.

Hung, S.-Y. (2003). Expert versus novice use of the executive support systems: An empirical study. Information \& Management, 40(3), 177-189.

Insko, C. A., Schopler, J., Graetz, K. A., Drigotas, S. M., Currey, D. P., Shannon, L. S. et al. (1994). Interindividual-intergroup discontinuity in the prisoner's dilemma game. The Journal of Conflict Resolution, 38(1), 87-116.

Johnson-Laird, P. N. (1988). The computer and the mind. Cambridge: Harvard University Press.

Kahneman, D. (2002). Maps of bounded rationality: A perspective on intuitive judgment and choice. In Nobel Prize Lectures. Princeton: Princeton University Press.

Kahneman, D. (2003a). Maps of bounded rationality: Psychology for behavioral economics. The American Economic Review, 93(5), 1449-1475.

Kahneman, D. (2003b). A psychological perspective on economics. The American Economic Review, 93(2), 162-168.

Kahneman, D., \& Tversky, A. (1973). On the psychology of prediction. Psychological Review, 80(4), 237-251.

Kahneman, D., \& Tversky, A. (1979). Prospect theory: An analysis of decision under risk. Econometrica, 47(2), 263-291.

Kendall, M. G., Bernoulli, D., Allen, C. G., \& Euler, L. (1961). Studies in the history of probability and statistics: XI. Daniel Bernoulli on Maximum Likelihood. Biometrika, 48(1/2), 1-18. 
Killingback, T., \& Doebeli, M. (2002). The continuous prisoner's dilemma and the evolution of cooperation through reciprocal altruism with variable investment. The American Naturalist, 160(4), 421-438.

Knight, F. (1921). Uncertainty and profit. Boston: Houghton Mifflin.

Kollock, P. (1998). Social dilemmas: The anatomy of cooperation. Annual Review of Sociology, 24, 183-214.

Kringelbach, M. L., \& Rolls, E. T. (2004). The functional neuroanatomy of the human orbitofrontal cortex: Evidence from neuroimaging and neuropsychology. Progress in Neurobiology, 72(5), 341-372.

Lang, B. (1964). Intuition in bloomsbury. Journal of the History of Ideas, 25(2), 295-302.

Leskowitz, E. (2002). Medical intuition. In S. Scott (Ed.), Handbook of complementary and alternative therapies in mental health (pp. 269-286). San Diego: Academic Press.

Lustrek, M., Gams, M., \& Bratko, I. (2006). Is real-valued minimax pathological? Artificial Intelligence, 170(6-7), 620-642.

Maia, T. V., \& McClelland, J. L. (2004). A reexamination of the evidence for the somatic marker hypothesis: What participants really know in the lowa gambling task. Proceedings of the National Academy of Sciences of the United States of America, 101(45), 16075-16080.

Maia, T. V., \& McClelland, J. L. (2005). The somatic marker hypothesis: Still many questions but no answers: Response to Bechara et al. Trends in Cognitive Sciences, 9(4), 162-164.

Mas-Colell, A., Whinston, D. M., \& Green, J. R. (1995). Microeconomic theory. Oxford: Oxford University Press.

Merleau-Ponty, M. (1962). Phenomenology of perception. London: Routledge \& Kegan Paul.

Mill, J. S. (1991). Collected works of John Stuart Mill (Vol. 2). London: Routledge and Kegan Paul. (Trabalho original publicado em 1963)

Nash, J. (1951). Non-cooperative games. The Annals of Mathematics, 54(2), 286-295.

Newton, P. S. (1941). Intuition and the traditional problems of philosophy. The Philosophical Review, 50(4), 396-409. 
O'Doherty, J., Kringelbach, M., Rolls, E., Hornak, J., \& Andrews, C. (2000). Medial and lateral orbitofrontal cortex differentially activated by reward and punishment during an emotion-related reversal task. Neurolmage, 11(5, Suppl. 1), S234-S234.

Pacini, R., \& Epstein, S. (1999). The relation of rational and experiential information processing styles to personality, basic beliefs, and the ratio-bias phenomenon. Journal of Personality and Social Psychology, 76(6), 972-987.

Payne, J. W., Bettman, J. R., \& Johnson, E. J. (1993). The adaptive decision maker. Cambridge: Cambridge University Press.

Perkins, D. N. (1977). The limits of intuition. Leonardo, 10(2), 119-125.

Pretz, J. E., \& Totz, K. S. (2007). Measuring individual differences in affective, heuristic, and holistic intuition. Personality and Individual Differences, 43(5), 1247-1257.

Rabin, M. (2002). Inference by believers in the law of small numbers. The Quarterly Journal of Economics, 117(3), 775-816.

Rapoport, A., \& Chammah, A. M. (1965). Prisoner's dilemma. Ann Arbor, MI: University of Michigan Press.

Ritchie, W. J., Kolodinsky, R. W., \& Eastwood, K. (2007). Does executive intuition matter? An Empirical Analysis of Its Relationship With Nonprofit Organization Financial Performance. Nonprofit and Voluntary Sector Quarterly, 36(1), 140-155.

Robert, M. E. (1993). Framing: Toward clarification of a fractured paradigm. The Journal of Communication, 43(4), 51-58.

Rolls, E. T. (2004). The functions of the orbitofrontal cortex. Brain and Cognition, 55(1), 11-29.

Rolls, E. T. (2006). Brain mechanisms of emotion and decision-making. International Congress Series, 1291, 3-13.

Sadikov, A., Bratko, I., \& Kononenko, I. (2005). Bias and pathology in minimax search. Theoretical Computer Science, 349(2), 268-281.

Sanfey, A. G., \& Cohen, J.D. (2004). Is knowing always feeling? Proceedings of the National Academy of Sciences of the United States of America, 101(48), 16709-16710.

Shiloh, S., Salton, E., \& Sharabi, D. (2002). Individual differences in rational and intuitive thinking styles as predictors of heuristic responses and framing effects. Personality and Individual Differences, 32(3), 415-429. 
Thaler, R. H., Tversky, A., Kahneman, D., \& Schwartz, A. (1997). The effect of myopia and loss aversion on risk taking: An experimental test. The Quarterly Journal of Economics, 112(2), 647-661.

Tilmann Betsch, G.-M. B. C. E. A. M. (1998). Natural sampling and base-rate neglect. European Journal of Social Psychology, 28(2), 269-273.

Turnbull, O. H., Evans, C. S., Bunce, A., \& Carzolio, B. (2005). Motion-based learning and central executive resourses: An investigation of intuition on the lowa Gambling Task. Brain and Cognition, 57(3), 244-247.

Tversky, A., \& Kahneman, D. (1974). Judgment under uncertainty: Heuristics and biases. Science, 185(4157), 1124-1131.

Tversky, A., \& Kahneman, D. (1981). The framing of decisions and the psychology of choice. Science, 211(4481), 453-458.

Tversky, A., \& Kahneman, D. (1982). Judgment under uncertainty: Heuristics and biases. In D. Kahneman, P. Slovic \& A. Tversky (Eds.), Judgment under uncertainty: Heuristics and biases (pp. 3-20). Cambridge: Cambridge University Press.

Tversky, A., \& Kahneman, D. (1986). Rational choice and the framing of decisions. The Journal of Business, 59(s4), S251.

Tversky, A., \& Kahneman, D. (1991). Loss aversion in riskless choice: A referencedependent model. The Quarterly Journal of Economics, 106(4), 1039-1061.

Tversky, A., Slovic, P., \& Kahneman, D. (1990). The causes of preference reversal. The American Economic Review, 80(1), 204-217.

Von Neumann, J., \& Morgenstern, O. (1944). Theory of games and economic behavior. Princeton: Princeton University Press.

Wansink, B., Kent, R. J., \& Hoch, S. J. (1998). An anchoring and adjustment model of purchase quantity decisions. Journal of Marketing Research, 35(1), 71-81.

Yoeli, R., Manor, G., \& Gill, A. (1989). Modeling human intuition in an expert system for planning aerial application operations. Computers and Electronics in Agriculture, 4(1), 13-22.

Recebido em: 4/08/2008

Aceito em: 9/02/2009

Álvaro Machado Dias, PhD, Pos-doc do LIM-21, Laboratório de Neuroimagem,

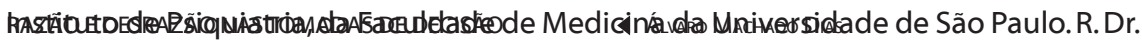
Ovidio Pires de Campos s/n. Endereço eletrônico: alvaromd@usp.br 